\title{
A Discussion on the Innovation Distribution of Markov Regime-Switching GARCH Model
}

\author{
$\underline{\text { Y.Shi }}^{\mathrm{a}}$ and K-Y. Ho ${ }^{\mathrm{a}}$ \\ ${ }^{a}$ Research School of Finance, Actuarial Studies and Statistics, The Australian National University, Canberra, \\ Australia \\ Email: yanlin.shi@anu.edu.au
}

\begin{abstract}
Markov Regime-Switching Generalized Autoregressive Conditional Heteroskedastic (MRSGARCH) model is a widely used approach to model the financial volatility with potential structural breaks. The original innovation of the MRS-GARCH model is assumed to follow the Normal distribution, which cannot accommodate fat-tailed properties commonly existing in financial time series. Many existing studies point out that this problem can lead to inconsistent estimates. To overcome it, the Student's t-distribution and General Error Distribution (GED) are the two most popular alternatives. However, a recent study points out that the Student's t-distribution lacks stability. Instead, this research incorporates the $\alpha$-stable distribution in the GARCH-type model. The issue of the $\alpha$-stable distribution is that its second moment does not exist. To solve this problem, the tempered stable distribution, which retains most characteristics of the $\alpha$-stable distribution and has defined moments, is a natural candidate. In this paper, we conduct a series of simulation studies to demonstrate that MRS-GARCH model with tempered stable distribution consistently outperform that with Student's t-distribution and GED. Our empirical study on the S\&P 500 daily return volatility also generates robust results. Therefore, we argue that the tempered stable distribution could be a widely useful tool for modelling the financial volatility in general contexts with a MRS-GARCH-type specification.
\end{abstract}

Keywords: GARCH Model, regime-switching, fat-tailed distribution, tempered stable distribution 


\section{INTRODUCTION}

The Generalized Autoregressive Conditional Heteroskedastic (GARCH) model proposed by Bollerslev (1986) can successfully capture many attractive characteristics of financial time series like time-varying heteroskedasticity and volatility clustering (Ho et al., 2013). Despite its popularity, the major problem of the original GARCH model is that it assumes that the conditional volatility has only one regime over the entire period. Hence, when structural breaks are present, it is argued that GARCH model always implies a high volatility persistence (Klaassen, 2002).

Hamilton (1989) proposes the Markov Regime-Switching (MRS) model to allow parameters to transit between state spaces. Based on his work, Haas et al. (2004) incorporate the MRS specification into the GARCH framework and propose the MRS-GARCH model. Originally, the innovation sequence is assumed to follow the Normal (Gaussian) distribution.

However, significant evidence suggests that the financial time series is rarely Gaussian but typically leptokurtic and exhibits fat-tail behaviour (Bollerslev, 1987). For the original GARCH model, (Bollerslev and Wooldridge, 1992) propose the Quasi Maximum Likelihood Estimation (QMLE), which can accommodate for fat-tailedness through their specification and still generates consistent estimates. For the MRS-GARCH model, however, if regimes are not Gaussian but leptokurtic, the use of within-regime normality can seriously affect the identification of the regime process (Klaassen, 2002). Haas and Paolella (2012) further argue that QMLE of the MRS-GARCH model is not consistent.

To solve this problem, a common solution is to employ a fat-tailed distribution such as the Student's tdistribution or General Error Distribution (GED) (Haas et al., 2004). However, a recent study by Calzolari et al. (2014) argues that the widely used Student's t-distribution is problematic. Its most serious drawback is that this distribution lacks in stability under aggregation, which is of particular importance in portfolio applications and risk management in finance study. As a replacement of the Student's t, the $\alpha$-stable distribution is recommended by Calzolari et al. (2014) to model the fat-tailed behaviour of financial time series for the original GARCH model. Additionally, they argue that similar to the Student's t, $\alpha$-stable distribution can be easily adapted to account for many characteristics of volatility such as asymmetry in the underlying financial time series. Unfortunately, since the second moment of the $\alpha$-stable distribution does not exist in most cases, GARCH-type model with this distribution will lead to problematic interpretation. Hence, the sought of an alternative distribution would be of particular interest for the application of GARCH-type model.

The tempered stable distribution is a natural substitution of the $\alpha$-stable. Firstly introduced in Koponen (1995), tempered stable distribution covers several well-known subclasses like Variance Gamma, bilateral Gamma and CGMY distributions (Küchler and Tappe, 2013). The advantage of it is that this distribution retains most of the attractive properties of the $\alpha$-stable distribution and has defined moments.

In this paper, we employ the MRS-GARCH model with the tempered stable distribution and argue that it outperforms the commonly used Student's $t$ and GED. To demonstrate that, we conduct a series of simulation studies to compare the performance of MRS-GARCH model with those three distributions. First, we set the true distribution as Student's $t$ and GED, respectively. Via twelve combinations of different transition probabilities and GARCH parameters, MRS-GARCH models with three distinct fat-tailed distributions are systematically analyzed. It is demonstrated that when the true distribution is Student's t or GED, the MRSGARCH model with tempered stable distribution generates very similar results as the true model. More importantly, it outperforms the other competitor in terms of consistency, efficiency and overall performance. Second, we let the tempered stable be the true distribution. Twelve sets of simulations are further constructed, including different choices of transition probabilities and tempered stable distribution parameters. In this scenario, either the Student's t or GED cannot perform as well as the tempered stable. Consequently, we argue that the tempered stable distribution could be a widely useful tool for modelling the financial volatility in general contexts with a MRS-GARCH-type specification.

To empirically compare the MRS-GARCH model with different distributions, we apply them to the daily return of the S\&P 500 index. The results suggest that the MRS-GARCH model with tempered stable distribution outperforms that with all the other distributions. Besides, the estimated parameters across MRS-GARCH with different distributions are relatively close to each other. Further, the density of the fitted tempered stable distribution is closer to that of the standardized data.

The remainder of this paper proceeds as follows. Section 2 describes the specification of the MRS-GARCH model employed in this study. Section 3 explains how the tempered stable distribution can be applied to the 
MRS-GARCH model. We conduct three independent simulation studies in Section 4. The empirical results are discussed in Section 5. Section 6 concludes the paper.

\section{MRS-GARCH MODEL}

In this paper, we employ the two-state MRS-GARCH(1,1) model investigated in Haas et al. (2004), and its specification is as follows:

$$
\begin{aligned}
r_{t} & =\mu+\varepsilon_{t} \text { and } \varepsilon_{t}=\eta_{t} \sqrt{h_{s_{t}, t}} \\
h_{s_{t}, t} & =\left\{\begin{array}{l}
\omega_{1}+\alpha_{1} \varepsilon_{t-1}^{2}+\beta_{1} h_{1, t-1} \text { when } s_{t}=1 \\
\omega_{2}+\alpha_{2} \varepsilon_{t-1}^{2}+\beta_{2} h_{2, t-1} \text { when } s_{t}=2
\end{array}\right.
\end{aligned}
$$

where $s_{t}$ is the state that $r_{t}$ lies in at time $t, h_{s_{t}, t}$ is the conditional volatility in state $s_{t}$ at time $t$ and $\eta_{t}$ is an identical and independent innovation sequence. In addition, the sequence $\left\{s_{t}\right\}$ is assumed to be a stationary, irreducible and latent Markov process with discrete state space $\{1,2\}$ and transition matrix $P_{i}=\left[p_{j k}\right]$ where $p_{j k}=P\left(s_{t+1}=k \mid s_{t}=j\right)$ is the one-step transition probability of moving from state $j$ to state $k(j, k \in$ $\{1,2\})$. The benefit of this specification is that the volatility persistence in state $j$ is measured by $P_{j}=\alpha_{j}+\beta_{j}$, as in the GARCH model (Haas et al., 2004). Also, we constrain that $\omega_{1} /\left(1-P_{1}\right) \omega_{2} /\left(1-P_{2}\right)$, so that the unconditional volatility of state 1 (calm state) is less than that in state 2 (turbulent state).

\section{Tempered Stable Distribution}

Although $\eta_{t}$ of the MRS-GARCH model is assumed to be Gaussian, due to the reason discussed in the introduction, a fat-tailed distribution like Student's t or GED should be employed instead (Haas and Paolella, 2012). However, Calzolari et al. (2014) suggests that the widely used Student's t-distribution lacks the stability-underaddition property. Further, they suggest that the $\alpha$-stable distribution can overcome this problem and should be employed as a replacement. Despite its attractive characteristics, the second moment of the $\alpha$-stable distribution does not exist in most cases. Consequently, the interpretation of conditional volatility would fail.

Alternative to the $\alpha$-stable, we suggest that the tempered stable distribution can be employed. A general case of the tempered stable distribution is characterized by six parameters and denoted as $T S\left(\alpha^{+}, C^{+}, \lambda^{+} ; \alpha^{-}, C^{-}, \lambda^{-}\right)$. The levy measure of such random variable $x$ is

$$
\nu(x)=\frac{c^{-}}{|x|^{1+\alpha^{-}}} e^{-\lambda^{-}|x|} 1_{x<0}+\frac{c^{+}}{|x|^{1+\alpha^{+}}} e^{-\lambda^{+}|x|} 1_{x>0}
$$

Therefore, a tempered stable distribution with zero mean has the following characteristic function (Cont and Tankov, 2004).

$$
\begin{array}{r}
\phi(t)=\exp \left\{\Gamma\left(-\alpha^{+}\right)\left(\lambda^{+}\right)^{\alpha^{+}} C^{+}\left[\left(1-\frac{i t}{\lambda^{+}}\right)^{\alpha^{+}}-1+\frac{i t \alpha^{+}}{\lambda^{+}}\right]\right. \\
\left.+\Gamma\left(-\alpha^{-}\right)\left(\lambda^{-}\right)^{\alpha^{-}} C^{-}\left[\left(1-\frac{i t}{\lambda^{-}}\right)^{\alpha^{-}}-1+\frac{i t \alpha^{-}}{\lambda^{-}}\right]\right\}
\end{array}
$$

where $\alpha^{+}, \alpha^{-}<2$ and $C^{+}, C^{-}, \lambda^{+}, \lambda^{-}>0$. Its first four cumulants are hereby defined as:

$$
\begin{aligned}
& \kappa_{1}=0 \\
& \kappa_{2}=\Gamma\left(2-\alpha^{+}\right) C^{+}\left(\lambda^{+}\right)^{\alpha^{+}-2}+\Gamma\left(2-\alpha^{-}\right) C^{-}\left(\lambda^{-}\right)^{\alpha^{-}-2} \\
& \kappa_{3}=\Gamma\left(3-\alpha^{+}\right) C^{+}\left(\lambda^{+}\right)^{\alpha^{+}-3}+\Gamma\left(3-\alpha^{-}\right) C^{-}\left(\lambda^{-}\right)^{\alpha^{-}-3} \\
& \kappa_{4}=\Gamma\left(4-\alpha^{+}\right) C^{+}\left(\lambda^{+}\right)^{\alpha^{+}-4}+\Gamma\left(4-\alpha^{-}\right) C^{-}\left(\lambda^{-}\right)^{\alpha^{-}-4}
\end{aligned}
$$

Hence, the first four moments of $x$ can be found via the following relations:

$$
\begin{aligned}
& m_{1}=\kappa_{1} \\
& m_{2}=\kappa_{2}+\kappa_{1}^{2} \\
& m_{3}=\kappa_{3}+3 \kappa_{2} \kappa_{1}+\kappa_{1}^{3} \\
& m_{4}=\kappa_{4}+4 \kappa_{3} \kappa_{1}+3 \kappa_{2}^{2}+6 \kappa_{2} \kappa_{1}^{2}+\kappa_{1}^{4}
\end{aligned}
$$


Therefore, it is clear that the tempered stable distribution has defined moments, so that it can be further applied to describe the innovation of GARCH-type model.

To apply this distribution into the MRS-GARCH model, we require $x$ to be standardized. Bianchi et al. (2010) argues that one way to achieve the standardization is letting

$$
C^{+}=\frac{p\left(\lambda^{+}\right)^{2-\alpha^{+}}}{\Gamma\left(2-\alpha^{+}\right)} \text {and } C^{-}=\frac{(1-p)\left(\lambda^{-}\right)^{2-\alpha^{-}}}{\Gamma\left(2-\alpha^{-}\right)}
$$

where $p \in(0,1)$, then $x \sim T S\left(\alpha^{+}, \lambda^{+}, \alpha^{-}, \lambda^{-}, p\right)$ has zero mean and unit variance.

Combining equations (3) and (6), we now have a standardized tempered stable distribution. To incorporate it into the MRS-GARCH model, the maximum likelihood estimation (MLE) procedure described in Haas et al. (2004) can be performed.

\section{Comparisons between Distributions: Simulation Studies}

We conduct three simulation studies to compare the performance of Student's t, GED and tempered stable distributions. ${ }^{1}$ The data generation process is MRS-GARCH(1,1) as described in equation (1) in all cases. True distributions of the three studies are therefore Student's t, GED and tempered stable, respectively. Altogether, twelve sets of simulations of the MRS-GARCH(1,1) process with different $p_{11}, p_{22}, P_{1}$ and $P_{2}$ are generated, where $\mu=0, \omega_{1}=0.1, \omega_{2}=0.5$, sample size is 5000 and the number of replicates for each set is 300 .

Comparing the simulation results, the following conclusions can be derived. In general, when the true distribution is Student's t or GED, MRS-GARCH with tempered stable distribution (MRS-GARCH-S) consistently outperforms (comparing the root-mean-squared-error) that with GED (MRS-GARCH-G) or Student's t (MRSGARCH-t), respectively. Also, the results of MRS-GARCH-S and those of the true model are very close in most situations. Nevertheless, MRS-GARCH-S can even lead to larger likelihoods than the true model. When the true distribution is the tempered stable, MRS-GARCH-t or MRS-GARCH-G cannot perform as well as the MRS-GARCH-S. All the above observations are robust across different combinations of transition probabilities and volatility persistence. We hereby argue that for a given financial time series with an unknown fat-tailed distribution of innovation, MRS-GARCH-S model should be employed to study its regime-switching conditional volatility.

\section{EMpirical RESUlts}

To empirically compare MRS-GARCH models with Student's t, GED and tempered stable distributions, we work on the dataset of the daily S\&P 500 Index (SP500). The daily closing prices for SP500 over the period from 1 January 2004 to 31 December 2013 are obtained from the Thomson Reuters Tick History (TRTH) database, which contains microsecond-time-stamped tick data dating back to January 1996. The database covers 35 million OTC and exchange-traded instruments worldwide, which are provided by the Securities Industry Research Centre of Australasia (SIRCA). The corresponding return in the percentage series is defined as the logarithm of the daily closing price differences times 100 ; that is, $r_{t}=100 \times \log \left(S_{t} / S_{t-1}\right)$.
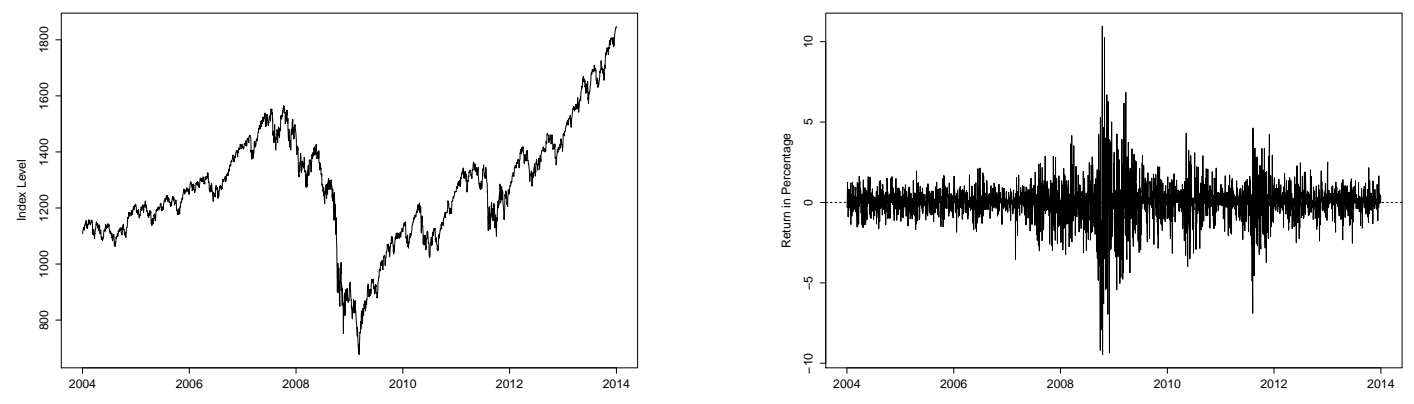

Figure 1. S\&P 500 Daily Index and Return.

${ }^{\mathrm{T}}$ Due to the page limit, the tables and description of this section are not presented here and are available upon request. 
The level and return of SP500 are plotted in Figure 1. In the level plot, SP500 level clearly decreases between 2008 and 2009, which is the global financial crisis (GFC) period. Demonstrated in the return plot, from 2008 to 2010 , the return becomes much more volatile. After 2010 , the volatility tends to be smaller, with some turbulence around the end of 2010 and the beginning of 2012. Hence, with the presence of GFC and preliminary visual evidence, structural breaks may possibly exist in the SP500 return. Consequently, as discussed in Section 2.2, MRS-GARCH model should be employed to study its volatility behaviour instead of the GARCH model. In addition, the mean and standard error of the SP500 return are 0.0202 and 1.2876 , respectively. The skewness is -0.3259 , indicating that the SP500 return is slightly negatively skewed. The kurtosis is 11.0235 , suggesting a non-Gaussian distribution. Thus, we perform the Kolmogorov-Smirnov and Jarque-Bera normality tests (not presented), where the null hypotheses indicating normality are rejected in both cases ( $\mathrm{p}$-values are 0.0000). As a result, as discussed in Section 3, the innovation of MRS-GARCH model should follow a fat-tailed distribution, rather than the Normal.

Table 1. Empirical Results: S\&P 500 Index.

\begin{tabular}{ccccccccc}
\hline & $p_{11}$ & $p_{22}$ & $\alpha_{1}$ & $\alpha_{2}$ & $\beta_{1}$ & $\beta_{2}$ & $\log . l i k$ & AIC \\
\hline Student's t & 0.9967 & 0.9936 & 0.0611 & 0.0877 & 0.8464 & 0.9035 & -3409 & 6839 \\
& $(0.0000)$ & $(0.0000)$ & $(0.0020)$ & $(0.0000)$ & $(0.0000)$ & $(0.0000)$ & & \\
GED & 0.9993 & 0.9987 & 0.0560 & 0.1064 & 0.8564 & 0.8879 & -3395 & 6813 \\
& $(0.0000)$ & $(0.0000)$ & $(0.0620)$ & $(0.0000)$ & $(0.0000)$ & $(0.0000)$ & & \\
Tempered Stable & 0.9992 & 0.9989 & 0.0613 & 0.1072 & 0.8985 & 0.8877 & -3382 & 6793 \\
& $(0.0000)$ & $(0.0000)$ & $(0.0310)$ & $(0.0000)$ & $(0.0000)$ & $(0.0000)$ & & \\
\hline
\end{tabular}

We fit the MRS-GARCH model with Student's t, GED and tempered stable distributions for the SP500 return. The results are presented in Table 1. Overall, all estimates are significant at 5\% level in all models, and estimates of individual parameter from different models are quite close. Specifically, both $p_{11}$ and $p_{22}$ are greater than 0.99 in all models, suggesting that the expectations of staying at both the calm and turbulent states are quite long. $\alpha_{1}+\beta_{1}$ is at around 0.92 , and $\alpha_{2}+\beta_{2}$ is at around 0.99 . This indicates that volatility of SP500 return is remarkably persistent over the entire period in both states. Also, a shock will affect the volatility longer in the turbulent state than in the calm state. To compare the model performance, both log-likelihood and Akaike Information Criterion (AIC) are presented. It can be seen that MRS-GARCH-S is preferred to the other two specifications and MRS-GARCH-G outperforms MRS-GARCH-t.
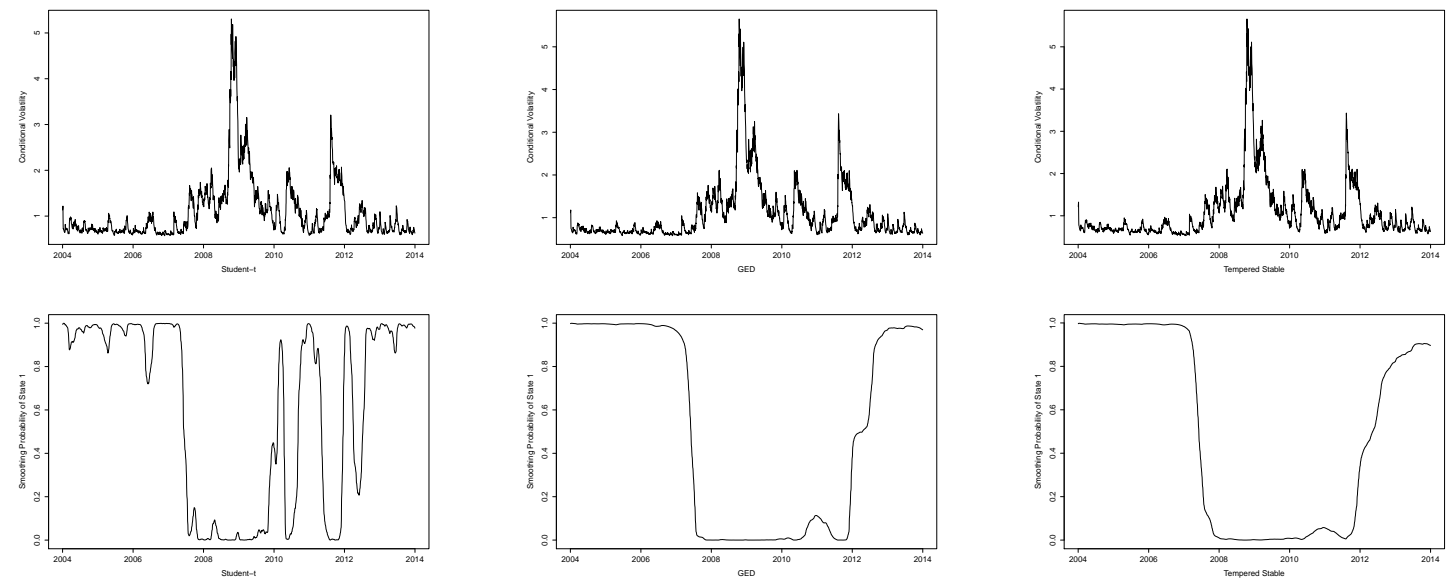

Figure 2. Fitted Conditional Volatility and Smoothing Probability. 
All the three estimated conditional volatility series ${ }^{2}$ and the smoothing probability of the calm state are plotted in Figure 2. Despite of their different model performance, the three estimated conditional volatility series have similar shapes. It could be due to the similarity of estimates. The trends of those series are consistent with our observation of the return series, since the conditional volatility is comparatively larger from 2008 to 2010. According to the smoothing probability, MRS-GARCH-t suggests SP500 return starts with the calm state and switches to the turbulent state from 2008. It stays there until the end of 2010, with a short jump back to the calm state. After that, SP500 return switches back to the calm state and stays there for most of the time, except for two short periods (the end of 2011 and mid-2012) back to the turbulent state. The results of both MRS-GARCH-G and MRS-GARCH-S are very similar and different from that of MRS-GARCH-t to some extent. Basically, they suggest SP500 return lies in the turbulent state from 2008 to 2011 and lies in the calm state during the other periods. Despite this difference, the three-state structure is largely concordant with the real macro-economic situation: the 2008 GFC with an effect lasting for around 3 years.

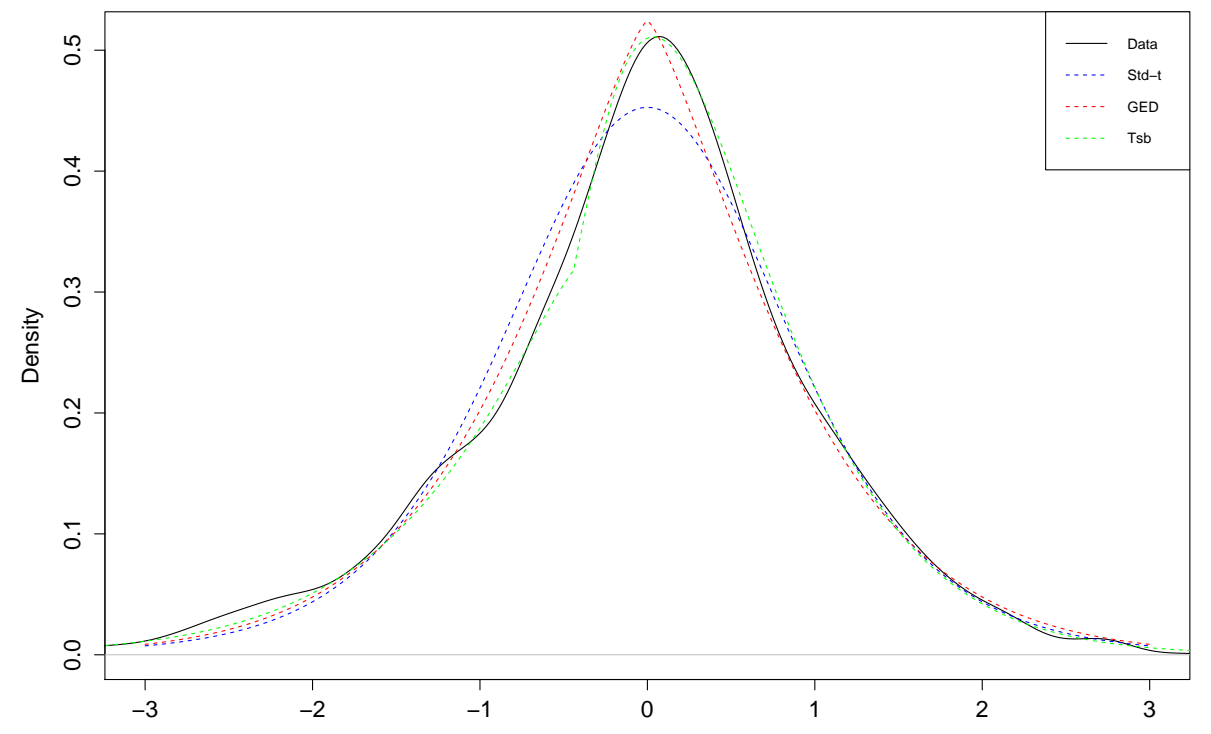

Figure 3. Density Plots of S\&P 500 Return and Choices of Distributions.

To further explore the difference between models, we report the smoothed density plot of the standardized SP500 return in Figure 3. To compare the fitness of the distributions, we also plot the densities of Student's $t$ and GED distributions with 7.2742 and 1.3301 degrees of freedom, respectively. Those are estimates from the fitted MRS-GARCH-t and MRS-GARCH-G models. The density of the fitted tempered stable distribution with parameters $\hat{a}_{1}=-0.1477, \hat{a}_{2}=-1.8164, \hat{\lambda}_{1}=2.7876, \hat{\lambda}_{2}=23.1898$ and $p=0.4407$ is also reported. It is clear that the shape of the tempered stable density is closer to that of the standardized SP500 return than the others. This provides further evidence that the MRS-GARCH-S outperforms the other models for our dataset.

\section{Conclusion}

MRS-GARCH model has enjoyed particular popularity in the financial study with the presence of structural breaks. However, the original MRS-GARCH model is based on the Normal distribution, and its estimates will be inconsistent for fat-tail-distributed data. As the financial data in practice are rarely Gaussian, the sought of an appropriate distribution to accommodate their excess kurtosis becomes an essential issue for the application of the MRS-GARCH model. As widely used alternatives of the Normal distribution, the Student's $t$ and GED are the common choices in most existing literature. A recent study by Calzolari et al. (2014) points out that due to the instability under aggregation, the Student's $t$ is not an optimal choice. To overcome that,

\footnotetext{
${ }^{2}$ We report conditional volatility here as the square root of $h_{t}$, so that it has the same scale as $r_{t}$.
} 
the $\alpha$-stable distribution is introduced. However, the undefined second moment of it could lead to problematic interpretation in the GARCH-type model.

To solve this issue, this paper suggests that the tempered stable distribution should be used instead. The reason is that this distribution retains all the attractive properties of the $\alpha$-stable and has defined moments. Via three different simulation studies of the MRS-GARCH(1,1) process, we systematically demonstrate the appropriateness of the tempered stable distribution applied in the MRS-GARCH model. The first two studies assume that the true distributions are the Student's $t$ and GED, respectively. In those cases, results of MRSGARCH-S are close to those of the true models. Additionally, MRS-GARCH-S generally outperforms its competitor (the other model except the true model) in terms of consistency, efficiency and overall performance. We construct different combinations of the underlying tempered stable distribution in the third study. Our results suggest that either MRS-GARCH-t or MRS-GARCH-G cannot perform as well as the MRS-GARCHS model.

Finally, empirical evidence is further provided as the robustness check of our simulation results. We fit the daily return of the S\&P 500 index into the three MRS-GARCH models, respectively. Our results suggest that MRS-GARCH-S is still preferred to the others. The density of fitted tempered stable distribution is closer to that of the standardized data than the other two. Hence, we argue that the tempered stable distribution could be a widely useful tool for modelling the financial volatility in general contexts with a MRS-GARCH-type specification.

\section{REFERENCES}

Bianchi, M. L., S. T. Rachev, Y. S. Kim, and F. J. Fabozzi (2010). Tempered stable distributions and processes in finance: numerical analysis. In Mathematical and Statistical Methods for Actuarial Sciences and Finance, pp. 33-42. Italy: Springer.

Bollerslev, T. (1986). Generalized autoregressive conditional heteroskedasticity. Journal of Econometrics 31(3), 307-327.

Bollerslev, T. (1987). A conditional heteroskedastic time series model for speculative prices and rates of return. Review of Economics and Statistics 69(3), 542-547.

Bollerslev, T. and J. M. Wooldridge (1992). Quasi-maximum likelihood estimation and inference in dynamic models with time-varying covariances. Econometric reviews 11(2), 143-172.

Calzolari, G., R. Halbleib, and A. Parrini (2014). Estimating garch-type models with symmetric stable innovations: Indirect inference versus maximum likelihood. Computational Statistics \& Data Analysis 76, $158-171$.

Cont, R. and P. Tankov (2004). Financial Modelling with Jump Processes. United Kingdom: Chapman and Hall/CRS Press.

Haas, M., S. Mittnik, and M. S. Paolella (2004). A new approach to markov-switching garch models. Journal of Financial Econometrics 2(4), 493-530.

Haas, M. and M. S. Paolella (2012). Mixture and regime-switching garch models. In L. Bauwens, C. Hafner, and S. Laurent (Eds.), Handbook of Volatility Models and Their Applications, pp. 71-102. United Kingdom: Wiley.

Hamilton, J. D. (1989). A new approach to the economic analysis of nonstationary time series and the business cycle. Econometrica 57(2), 357-384.

Ho, K.-Y., Y. Shi, and Z. Zhang (2013). How does news sentiment impact asset volatility? evidence from long memory and regime-switching approaches. North American Journal of Economics and Finance 26, 436-456.

Klaassen, F. (2002). Improving garch volatility forecasts with regime-switching garch. Empirical Economics 27(2), 363-394.

Koponen, I. (1995). Analytic approach to the problem of convergence of truncated lévy flights towards the gaussian stochastic process. Physical Review E 52(1), 1197.

Küchler, U. and S. Tappe (2013). Tempered stable distributions and processes. Stochastic Processes and their Applications 123(12), 4256-4293. 